\title{
Declining Incidence of Nasopharyngeal Carcinoma in Brunei Darussalam: a Three Decade Study (1986-2014)
}

\author{
Vui Heng Chong1*, Pemsari Upali Telisinghe ${ }^{2}$, Edwin LIM², Muhammad Syafiq \\ Abdullah $^{1}$, Fidah Idris ${ }^{3}$, Chee Fui Chong ${ }^{4}$
}

\begin{abstract}
Background: Nasopharyngeal carcinoma (NPC) is linked to Epstein Barr virus infection and is particularly common in the Far East, particularly among some Chinese groups. Certain ethnicities have been reported to have low incidence of NPC. This study looked at NPC in Brunei Darussalam over a three decade period. Materials and Methods: The cancer registry from 1986 to 2014 maintained by the State Laboratory was retrospectively reviewed. The age standardized rates (ASR) and the age specific incidence rates (ASIR) were calculated. Non NPC tumors were excluded from the study. Results: Altogether, there were a total of 450 NPC cases diagnosed accounting for $4.4 \%$ of all total cancer cases over the study period, declining from $10.3 \%$ in $1986-1990$ to $2.3 \%$ in 2011-2014. The most common tumor type was the undifferentiated carcinoma $(96.4 \%)$. The case characteristics were mean age $50.4 \pm 14.4$ years old, male $69 \%$, and predominately Malays $74.4 \%$, followed by Chinese $16.7 \%$. The mean age of diagnosis increased over the study period from 45.6 \pm 17.1 years $(1986-1989)$ to $54.1 \pm 12.5$ years (ANOVA, $\mathbf{p}<\mathbf{0 . 0 1}$ for trend). There were no differences in the mean age of diagnosis between the ethnic groups or genders. The ASR showed a declining trend from 11.1 per 100,000 in 1986-1990 to 5.95 per 100,000 in 2011-2014, similar trends been observedfor both genders. Among the age groups, declining trends were seen in all the other age groups apart from the $>70$ years group. The overall ASRs for the Malays and Chinese were 7.92/100,000 and 8.83/100,000 respectively, both showing declining trends. Conclusions: The incidence of NPC in Brunei Darussalam is comparable to rates reported from Singapore and Malaysia, but higher than rates reported from the other Southeast Asian nations. Unlike higher rates reported for Chinese compared to the Malays in other countries, the rates between the Malays and Chinese in our study was comparable. Importantly, the ASR is declining overall and for both genders and ethnic groups.
\end{abstract}

Keywords: Nasopharyngeal neoplasm - incidence - trend - epidemiology - Brunei Darussalam

Asian Pac J Cancer Prev, 16 (16), 7097-7101

\section{Introduction}

Nasopharyngeal carcinoma (NPC) is a tumor that arises from the epithelium of the posterior nasopharynx and is the most common tumor of the upper aerodigestive tract. The underlying pathogenesis is influenced by a combination of factors; genetic (i.e. certain HLA types), environmental (i.e. nitrosamine containing food such as salted food, rancid butter, tobacco, wood and formaldehyde) and viral infection (Epstein Barr virusEBV) resulting in sequence of changes from dysplasia to carcinoma. (Chan et al., 2002; Wei and Sham, 2005; Tsao et al., 2014; Petersson, 2015) In 1978, the World Health Organization has classified NPC into three histological subtypes; squamous cell carcinoma (SCC) as WHO type 1 , non-keratinizing carcinoma (WHO type 2), and undifferentiated carcinoma (WHO type 3). In 1991, the use of numerical designation was eliminated and the tumor classified as keratinizing SCC and non-keratinizing carcinoma, the latter being subdivided into differentiated and undifferentiated. (Shanmugaratnam and Sobin., 1993) The current classification included an additional category; basaloid SCC (Chan et al., 2005).

NPC exhibit peculiar geographic and ethnic distributions. It is particularly common in the East. In 2012, the Western Pacific (WPRO) and South-East Asia (SEARO) region accounted for $53.3 \%$ and $24.4 \%$ respectively, of the estimated global NPC burden. (IARC 2012) The other WHO Member States regions, Africa $7.0 \%$, Europe $5.8 \%$, the Americas $4.7 \%$ and East Mediterranean $4.7 \%$ accounted for the rest (IARC 2012). Globally, it is estimated that there are annually 80,000 new cases of NPC with 50,000 deaths (Bray et al., 2005). Apart from South-East Asia, Southern China (Guangdong province) has reported some of the highest rates with annual incidence of $>20 / 100,000$. (Zhang et al., 2015) NPC is particularly common in certain ethnic races; especially Chinese and this correspond to the high 
Vui Heng Chong et al

rates reported in the South-East Asia and Southern China. Differences are also seen in the tumor types (Petersson F., 2015) and also the stage of disease (Ianovski et al., 2010) at presentations.

As the incidence of cancers is increasing worldwide, it is very important to be aware of the characteristics and incidence, including diseases trends at the individual country, regional and international levels. These will allow for effective prevention programs to be formulated that suite the individual country, at the same time in line with regional and international policies in diseases prevention. In our setting, there has only been one study that looked at NPC. (Yeo et al., 2012) This study which looked at EBV and NPC reported that the incidence in Chinese was lower than the Malay (Yeo et al., 2009). However, this study only looked at one time point and did not assess the trend. This present study assessed the trend in the incidence of NPC over a three decade periods (1986-2014) and also assessed the trends among the genders and the two major ethnic groups (Malays and Chinese) in Brunei Darussalam.

\section{Materials and Methods}

\section{Setting}

Brunei Darussalam is a small developing nation with a total area of $5,765 \mathrm{~km} 2$ and estimated population of 415,717 (July 2013; Department of Economic Planning, Ministry of Finance). The population breakdown consists of ethnic group (Malays $66.3 \%$, ethnic Chinese $11 \%$ and indigenous $2.7 \%$ ) and the expatriate group (20\%).

\section{Data Source}

The cancer registry maintained by the Department of Pathology were retrospectively review from 1986-2014.In this registry, only histology proven cancers are registered. All biopsies specimen are sent to the State pathology Laboratory (located in the only government tertiary hospital, RIPAS, Bandar Seri Begawan) for analyses. Being the only state histopathology laboratory, the registry capture all the tissue diagnoses NPC cases for the whole country and is therefore representative.

For this study only NPC (WHO Classification) were studied. (Chan et al., 2005) Based on this classification; NPC is classified into Nasopharyngeal tumors not within this classification were excluded. In total, 15 cases were excluded which included lymphoma $(n=13)$ and adenoid cystic tumor $(n=2)$.

\section{Statistic}

The data were entered into the Excel (Microsoft) and later transferred into the Statistical Package for Social Sciences (SPSS, Version 16.0, Chicago, IL USA) program for analysis. Data for 1990 were missing and this was excluded from analysis; calculation of the Age Standardized Rates (ASR) and Age Specific Incidence Rates (ASIR). Chi-squared test was used to compare the categorical variables and the ANOVA and MannWhitney non-parametric test were used to compare the continuous variables. A p value of less than 0.05 was taken as significant.

\section{Results}

Altogether, there were a total of 450 NPC cases diagnosed over the study period, accounting for $4.4 \%$ of all total cancer cases. The incidence rates among all cancers declined from $10.3 \%$ in $1986-1990$ to $2.3 \%$ in 2011-2014 (Figure 1).

The most common tumor type was the undifferentiated carcinoma, accounting for over $96.4 \%$ and the remainders were keratinizing squamous cell carcinoma (3.6\%)

There were more men (69\%) compared to women and the mean age of diagnosis was $50.4 \pm 14.4$ years old with a

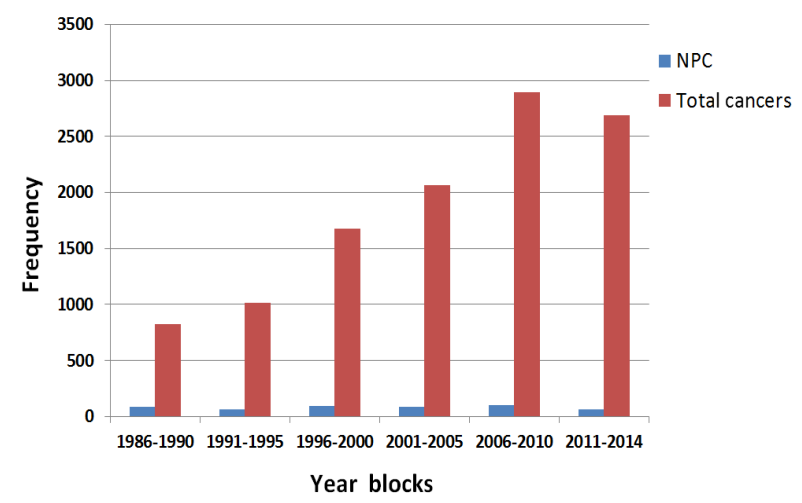

Figure 1. Proportion of Nasopharyngeal Carcinoma among All Cancer Cases Encountered

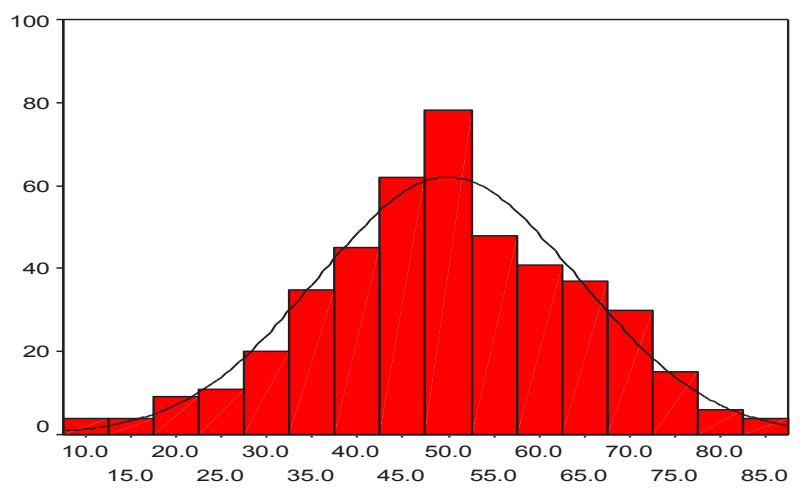

Figure 2. Age Distribution Showing Unimodal Distribution

Table 1. Mean Age of Diagnosis

\begin{tabular}{lcc}
\hline Variables & $\begin{array}{c}\text { Mean age } \\
\text { (Standard deviation) years }\end{array}$ & $\mathrm{p}$ values \\
\hline Genders & & \\
$\quad$ Men & $50.4 \pm 14.3$ & $\mathrm{p}>0.05$ \\
$\quad$ Women & $49.1 \pm 14.6$ & \\
Ethnicity & & \\
$\quad$ Malays & $50.7 \pm 14.6$ & 0.274 for trend \\
Chinese & $47.9 \pm 13.3$ & \\
Indigenous & $49.7 \pm 16.5$ & \\
Others & $44.7 \pm 8.1$ & \\
Years & & \\
1986-1989 & $45.6 \pm 17.1$ & $\mathrm{p}<0.001$ for trend \\
1991-1995 & $45.5 \pm 12.8$ & \\
1996-2000 & $50.7 \pm 13.4$ & \\
2001-2005 & $49.8 \pm 14.2$ & \\
2006-2010 & $52.9 \pm 14.6$ & \\
$2011-2014$ & $54.1 \pm 12.5$ & \\
\hline
\end{tabular}



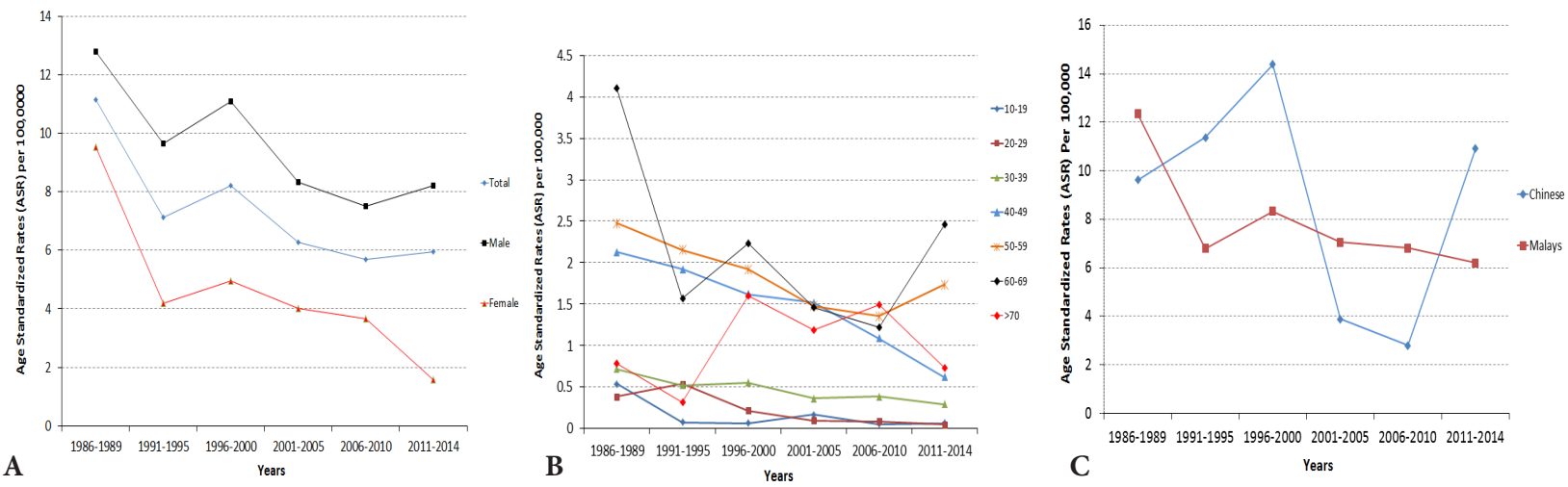

Figure 3.A) The Age Standardized Rates (ASR) for NPC Over the Study Period for Overall, Male and Female, B) the ASRs for the Different Age Groups, and C) the ASRs for the two Major Ethnic Groups (Malays and Chinese)
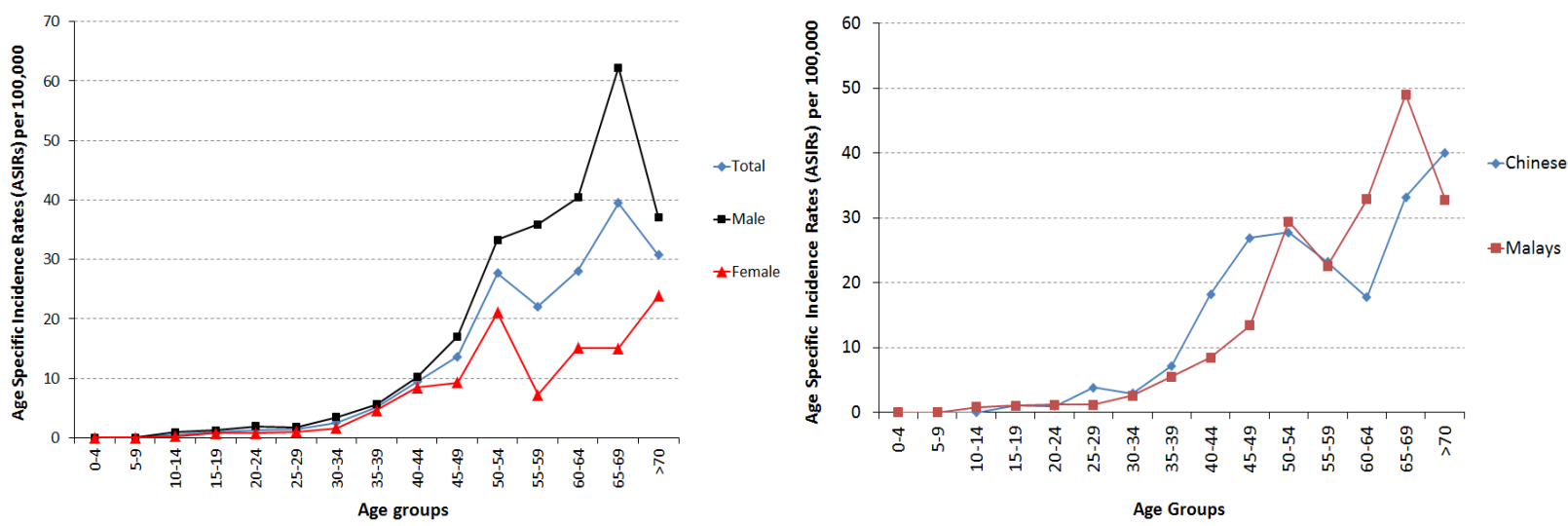

Figure 4. a) The Overall Age Specific Incidence Rates (ASIRs) for NPC; Overall, Male and Female, B) the ASIRs for the two Major Ethnic Groups (Malays and Chinese)

significant increasing trend seen over the period (ANOVA $\mathrm{p}<0.001)$. There was only one peak in the age distribution (Figure 2). Among the genders and the ethnic groups, there were no differences ( $\mathrm{p}>0.05)$ (Table 1).

The ASR showed declining trend from 11.13 in 19861989 to 5.95 per 100,000 in the latest time block (20112014) (Figure 3a). Declining trends were also among the genders; Male ASR 12.78/100,000 (1986-1990) to 8.2/100,000 (2010-2014) and Female ASR 9.52/100,000 (1986-1990) to $1.59 / 100,000$ (2010-2014). Apart from the $>70$ years age group, declining trends were seen in all the other age groups (Figure $3 b$ ).

The overall ASRs for the Malays and Chinese were $7.92 / 100,000$ and 8.83/100,000 respectively. There was a wide fluctuation for the Chinese and this likely due to the overall small population. For the Malays, the trend was declining with no fluctuation (Figure $3 \mathrm{c}$ ).

The ASIRs showed continued increase with fluctuation, peaking in the 65-69 age group. Similar trend was observed in the male but for the female group, the peak was in the $>70$ years group (Figure $4 \mathrm{a}$ ). The ASIRs for the two major ethnic groups were comparable (Figure $4 b$ ). The ASIRs for the Chinese were higher until the 50-54 years age groups, when the Malays were higher, until the $>70$ years age group.

\section{Discussion}

In our study, we showed that NPC accounted for $4.4 \%$ of all cancers encountered in our population over the three decade with a declining trend from $10.3 \%$ in 1986-1990 to $2.3 \%$ in $2011-2014$. Of importance is that the decline occurred in the background of increasing number of cancer cases seen each year. The total number of NPC cases had initially leveled off and in the later part of the study, actually declined. In Brunei, the continued increase of total cancers cases mainly due to increase in several cancers; colorectal cancer, breast and ovarian cancer.

The mean age of diagnosis of our population is comparable to what have been reported, most common in the 50-60 age groups. There was only one mode. There were no difference in the mean age of diagnosis between the genders and the ethnic groups. Importantly, between the time periods, the mean age of diagnosis increased significantly. This is likely due to effect of ageing population.

Similar to what have been reported in the literature, our study showed that NPC is more common among men, two to three times more common (Our study ASR male; female ratio was by a factor of 1.87 , Hawaiian (Caucasians 0.78, Chinese 2.4), Singapore (overall 2.81, Chinese 2.79, Malays 2.71) and Malaysia (overall 2.66, Chinese 2.45, Malays 2.83 and Indians 3.0). High incidence among men is probably related to exposure to higher risk factor such as smoking. Genetic differences (human leucocyte antigen, enzymes polymorphisms including DNA repair enzymes and susceptibility loci in chromosomes) have been shown to play a role in ethnic differences, but it is unknown if it 
play a role in gender differences. Other risk factors such as exposure to burning woods (i.e. cooking), consumption of salted food and exposure to EBV would be equally shared risk factors. Alcohol consumption (Chan et al., 2002; Wei and Sham, 2005; Chen et al., 2009) and occupational exposure to formaldehyde and wood or wood dust are also reported to be important factors (Hildesheim et al., 2001). However, in our setting, such exposures are not or now less common. Environmental factors are important as migrant studies have shown that the rates decline to intermediate rates between the countries of origin and countries of residence. (Yu and Hussain 2009; Mousavi et al., 2010). Low economic social status, an indirect indicator to exposures of the risk factors has also been reported to be associated with NPC. (Turkoz et al., 2011)

The predominant tumor type among our population is the undifferentiated carcinoma (previously known as WHO type 3 tumor), accounting for over $95 \%$ of all tumor. These findings are consistent with have been reported in other countries. (Wided et al., 2015) In Singapore, undifferentiated NPC accounts for $85.1 \%$ of all nasopharyngeal cancers. (Singapore Cancer Registry 2007) Of the three tumor types, undifferentiated NPC has been strongly linked to EBV infection (Raab-Traub. 2002) which serves as a driver for pathogenesis of this particular tumor type. (Chan et al., 2002)

NPC is known to vary between countries. In SouthEast Asia, the incidence of NPC vary between countries with higher rates in Malaysia (8th common cancer, ASR 7.2/100,000), Singapore (11th, 6.4/100,000), Indonesia (9th, 5.6/100,000) and Viet Nam (7th, 5.4/100,000) to lower rates in Myanmar (15th, 2.4/100,000), Philippines (15th, 2.2/100,000), Thailand (16th, 2.1/100,000), Cambodia (19th, 1.9/100,000), and the LAO republic $(17$ th, 1.7/100,000). Based on the IARC data, Brunei registered an ASR of 5.0/100,000 for NPC which placed it as the 12th most common cancers for that year. (IARC, 2012) The rate reported for 2011-2014 was 6.0/100,000 placing Brunei third in the South-East Asia region. Compared to the other parts of the world, the incidence are low with ASR of $<1.0 / 100,000$. (Jemal et al., 2011; IARC 2012) Outside of South-East Asia, the highest reported have come from Southern China (Guangdong province) with rates of $21.73 / 100,000$ (male 30.29/100,000 and female 13.09/100,000). (Zhang et al., 2015)

Of important note is that the rates have been decreasing trend. The ASR declined from a high of just over $11 / 100,000$ in $1986-1990$ period to just under $6 / 100,000$ in the latest period 2011-2014. The drop was rapid from 1986-1990 to 1991-1995 and then slowly leveled off after that. Interestingly, our overall ASRs for the two major ethnic groups were comparable; Malays $(7.92 / 100,000)$ and Chinese $(8.83 / 100,000)$. This is in contrast to the findings from neighboring countries, Singapore and Malaysia which have almost similar population ethnicities, apart from very few ethnic local Indians in Brunei Darussalam. The NPC rates are much higher among the Chinese in both Singapore (Male ASR 12.5/100,000) and Malaysia (Male 10.9/100,000, Female 3.9/100,000) compared to the Malays; Singapore (Male ASR 6.8/100,000) and Malaysia (Male 4.9/100,000). In both countries, the Indian had the lowest rates. (Singapore Cancer Registry 2007; Malaysia Cancer Registry 2007)

In the last three decades, the incidence rates of NPC have been declining among Chinese. These have been reported among Chinese residing in the South-East Asia, Hong Kong, Taiwan, and Singapore (Luo et al., 2007). Data from the Singapore cancer registry reported declining trends of NPC with an estimated annual decline of $0.96 \%$ for male and $1.89 \%$ for female in the period between 1968 and 2007. (Singapore Cancer Registry 2007) Declining trend has been reported from Guangzhou (Li et al., 2014), but not in Zhongshan. (Wei et al., 2010) Declining trends were also observed in our study for both Chinese and Malays. The decline was smooth among the Malays whereas there was wide fluctuation for the Chinese. This is likely accounted for by the overall small Chinese population in the country as even small fluctuations in the incident cases can lead to wide fluctuation in the calculated rates. Declining rate has also been reported in the northern Africa, Tunisia seen more in adult with annual average change of $-3.3 \%$ for men and $-2.7 \%$ for women. In the younger age group, the rates were stable. (Wided et al., 2015) Importantly, along with the declining incidence of NPC, the mortality rate has also declined. (Huang et al., 2012)

The declining rates observed in our studies, consistent with what have been generally reported elsewhere are accounted for by several important factors. In Brunei, the most important factor is perhaps the improvement in the standards of living; decline in reliance on firewood resulting in less exposure to smoke, consumption of less salted preserved meats and also consumption of more fresh produces (vegetables and fruits). The prevalence of tobacco use and exposure to EBV remain the same, but may still play a role in the decline in the incidence of NPC. Exposures to the latter two factors are now probably occurring at later age and hence may affect the NPC pathogenesis.

Declining in the incidence of NPC has implications in policy planning. Generally, we have observed that cancers linked to infections such as Helicobacter pylori and gastric cancer, Human papilloma virus (HPV) and cervix cancer and EBV and NPC are declining not just in Brunei but also other parts of the world. On the other hand cancers linked to non-communicable diseases especially obesity continues to increase. Cancers such as colorectal and breast cancers are increasing and these cancers are largely preventable. (IARC 2012) Screening programs such as colorectal cancer screening or screening mammography have been shown to be effective. Therefore, available resources that may be scarce in some countries should be targeted to the more common and increasing cancers. However, some resources should also be allocated cancers that are declining, including NPC for early detection, effective intervention and also prevention.

In conclusion, our study showed that the incidence of NPC was comparable to what have been reported in neighboring countries with almost similar population demographics. However, the rates between the two major ethnic groups (Malays and Chinese) were comparable, unlike rates reported in Singapore and Malays. Importantly, 
the incidence rates are declining, overall, for the genders and the Malays and Chinese.

\section{References}

Bray F, Haugen M, Moger TA, et al (2008). Age-incidence curves of nasopharyngeal carcinoma worldwide: bimodality in low-risk populations and aetiologic implications. Cancer Epidemiol Biomarkers Prev, 17, 2356-65.

Chan AT, Teo PM, Johnson PJ (2002). Nasopharyngeal carcinoma. Ann Oncol, 13, 1007-15.

Chan JK, Pilch BZ, Kuo TT, Wenig BM, Lee AW (2005). Tumours of the nasopharynx: introduction. in world health organization classificationof tumours. pathology and genetics. head and neck tumours. ed barnes 1, eveson jw, reichart p, sidransky D. IARC Press, Lyon 2005. Pg 83-4.

Chen L, Gallicchio L, Boyd-Lindsley K, et al (2009). Alcohol consumption and the risk of nasopharyngeal carcinoma: a systematic review. Nutr Cancer, 61, 1-15.

Hildesheim A, Dosemeci M, Chan CC, et al (2001). Occupational exposure to wood, formaldehyde, and solvents and risk of nasopharyngeal carcinoma. Cancer Epidemiol Biomarkers Prev, 10, 1145-53.

Huang TR, Zhang SW, Chen WQ, et al (2012). Trends in nasopharyngeal carcinoma mortality in China, 1973-2005. Asian Pac J Cancer Prev, 13, 2495-502.

Ianovski I, Izzard M, Morton RP, Plank LD (2010). Nasopharyngeal carcinoma: differences in presentation between different ethnicities in the New Zealand setting. ANZ J Surg, 80, 254-7.

International Agency for Research Centre. World Health Organisation. GLOBOCAN 2012: Estimated cancer incidence, mortality and prevalence worldwide in 2012. Retrieved from http://globocan.iarc.fr/old/pie_site.asp?sel ection $=18020 \&$ title $=$ Nasopharyn $x \&$ se $x=0 \&$ type $=0 \& p o p u$ lations $=1 \&$ window $=1 \&$ join $=1 \&$ submit $=\% \mathrm{C} 2 \% \mathrm{~A} 0$ Execut e\%C2\%A0 (Accessed 15th April 2015).

Jemal A, Bray F, Center MM, et al (2011). Global cancer statistics. CA: A Cancer J Clinicians, 61, 69-90.

Li K, Lin GZ, Shen JC, Zhou Q (2014). Time trends of nasopharyngeal carcinoma in urban Guangzhou over a 12-year period (2000-2011): declines in both incidence and mortality. Asian Pac J Cancer Prev, 15, 9899-903.

Luo J, Chia KS, Chia SE, et al (2007). Secular trends of nasopharyngeal carcinoma incidence in Singapore, Hong Kong and Los Angeles Chinese populations, 1973-1997. Eur J Epidemiol, 22, 513-21.

Ministry of Health Malaysia. National Cancer Registry Report 2007: Malaysia Cancer Statistics- Data and Figure 2007. Available from http://www.care.upm.edu.my/ dokumen/13603_NCR2007.pdf (Retrieved 16th June 2015).

Mousavi SM, Sundquist J, Hemminki K (2010). Nasopharyngeal and hypopharyngeal carcinoma risk among immigrants in Sweden. Int J Cancer, 127, 2888-92.

National Registry of Disease Office, Singapore. Singapore Cancer Registry Report No.7. Trends in cancer incidence in Singapore 1968-2007. Available from https://www. nrdo.gov.sg/docs/librariesprovider3/Publications-Cancer/ inc_report_v745ce06a5c9d76bafab5aff000014cdee. pdf?sfvrsn=0 (Accessed 16th June 2015)

Petersson F (2015). Nasopharyngeal carcinoma: a review. Semin Diagn Pathol, 32, 54-73.

Raab-Traub N (2002). Epstein-Barr virus in the pathogenesis of NPC. Semin Cancer Biol, 12, 431-441.

Shanmugaratnam K, Sobin LH (1993). The World Health Organization histological classification of tumours of the upper respiratory tract and ear. A commentary on the second edition. Cancer, 71, 2689-97.

Tsao SW, Yip YL, Tsang CM, et al (2014). Etiological factors of nasopharyngeal carcinoma. Oral Oncol, 50, 330-8.

Turkoz FP, Celenkoglu G, Dogu GG, et al (2011). Risk factors of nasopharyngeal carcinoma in Turkey-an epidemiological survey of the anatolian society of medical oncology. Asian Pac J Cancer Prev, 12, 3017-21.

Wei K, Xu Y, Liu J, Zhang W, Liang Z (2010). No incidence trends and no change in pathological proportions of nasopharyngeal carcinoma in Zhongshan in 1970-2007. Asian Pac J Cancer Prev, 11, 1595-9.

Wei WI, Sham JS (2005). Nasopharyngeal carcinoma. Lancet, 365, 2041-54.

Wided BA, Hamouda B, Hamadi H, Mansour BA (2015). Nasopharyngeal carcinoma incidence in North Tunisia: negative trends in adults but not adolescents, 1994-2006. Asian Pac J Cancer Prev, 16, 2653-7.

Yeo CH, Abdullah MS, Telesinghe PU, Ramasamy R (2009). Nasopharyngeal carcinoma in brunei darussalam: low incidence among the Chinese and an evaluation of antibodies to epstein-barr virus antigens as biomarkers. Singapore Med $J$, 50, 371-7.

Yu WM, Hussain SS (2009). Incidence of nasopharyngeal carcinoma in Chinese immigrants, compared with Chinese in China and South East Asia: review. J Laryngol Otol, 123, 1067-74.

Zhang LF, Li YH, Xie SH, et al (2015). Incidence trend of nasopharyngeal carcinoma from 1987 to 2011 in Sihui County, Guangdong Province, South China: an age-periodcohort analysis. Chin J Cancer, 34, 15. 